Acta Crystallographica Section E

Structure Reports

Online

ISSN 1600-5368

\section{Benzoylmethyl pyridine-4-carboxylate}

\section{Jian-Nan Guo, ${ }^{\mathrm{a} *}$ Bo-Chao Zhang, ${ }^{\mathrm{b}}$ Yi Jin, ${ }^{\mathrm{a}}$ Guo Tang ${ }^{\mathrm{a}}$ and Yu-Fen Zhao ${ }^{a}$}

${ }^{a}$ The Key Laboratory for Chemical Biology of Fujian Province, Department of Chemistry, Xiamen University, Xiamen 361005, People's Republic of China, and ${ }^{\mathbf{b}}$ Department of Chemistry, Xiamen University, Xiamen 361005, People's Republic of China

Correspondence e-mail: t12g21@xmu.edu.cn

Received 31 March 2008; accepted 11 May 2008

Key indicators: single-crystal X-ray study; $T=173 \mathrm{~K}$; mean $\sigma(\mathrm{C}-\mathrm{C})=0.003 \AA$; $R$ factor $=0.039 ; w R$ factor $=0.074 ;$ data-to-parameter ratio $=12.3$.

In the crystal structure of the title compound, $\mathrm{C}_{14} \mathrm{H}_{11} \mathrm{NO}_{3}$, isolated from the reaction of 2-bromo-1-phenylethanone and pyridine-4-carboxylic acid using triethylamine as a base to deprotonate the organic acid, the molecular packing is stabilized by $\mathrm{C}-\mathrm{H} \cdots \pi$ interactions involving the phenyl and pyridine rings. The $\mathrm{C}-\mathrm{C}-\mathrm{O}-\mathrm{C}$ torsion angle for the linkage between the two carbonyl groups is $-80.8(2)^{\circ}$, and the planes of the phenyl and pyridyl rings form a dihedral angle of $65.8(1)^{\circ}$.

\section{Related literature}

For related literature, see: Allen et al. (1987); Hendrickson \& Kandall (1970); Pavel et al. (1993).<smiles>O=C(COC(=O)c1ccncc1)c1ccccc1</smiles>

\section{Experimental}

Crystal data

$\mathrm{C}_{14} \mathrm{H}_{11} \mathrm{NO}_{3}$

$M_{r}=241.24$

Triclinic, $P \overline{1}$

$$
\begin{aligned}
& \alpha=106.738(7)^{\circ} \\
& \beta=114.495(8)^{\circ} \\
& \gamma=96.549(6)^{\circ} \\
& V=583.52(8) \AA^{3} \\
& Z=2
\end{aligned}
$$

Data collection

Bruker APEX CCD diffractometer Absorption correction: multi-scan (SADABS; Bruker, 2001)

$T_{\min }=0.971, T_{\max }=0.988$

\section{Refinement}

$R\left[F^{2}>2 \sigma\left(F^{2}\right)\right]=0.039$

$w R\left(F^{2}\right)=0.074$

$S=0.82$

2011 reflections

Mo $K \alpha$ radiation

$\mu=0.10 \mathrm{~mm}^{-1}$

$T=173(2) \mathrm{K}$

$0.30 \times 0.17 \times 0.12 \mathrm{~mm}$

4945 measured reflections 2011 independent reflections 1099 reflections with $I>2 \sigma(I)$ $R_{\text {int }}=0.042$

163 parameters

$\mathrm{H}$-atom parameters constrained

$\Delta \rho_{\max }=0.11{\mathrm{e} \AA^{-3}}^{-3}$

$\Delta \rho_{\min }=-0.13 \mathrm{e}^{-3}$

Table 1

Hydrogen-bond geometry $\left(\AA{ }^{\circ}\right)$.

\begin{tabular}{lllll}
\hline$D-\mathrm{H} \cdots A$ & $D-\mathrm{H}$ & $\mathrm{H} \cdots A$ & $D \cdots A$ & $D-\mathrm{H} \cdots A$ \\
\hline $\mathrm{C} 4-\mathrm{H} 4 \cdots \mathrm{CgB}^{\mathrm{i}}$ & 0.95 & 3.86 & $4.766(3)$ & 160 \\
$\mathrm{C} 2-\mathrm{H} 2 \cdots \mathrm{CgB}^{\mathrm{ii}}$ & 0.95 & 2.89 & $3.640(3)$ & 137 \\
$\mathrm{C} 6-\mathrm{H} 6 \cdots \mathrm{CgB}^{\text {iii }}$ & 0.95 & 3.00 & $3.752(3)$ & 137 \\
$\mathrm{C} 12-\mathrm{H} 12 \cdots \mathrm{CgA}^{\text {iv }}$ & 0.95 & 2.88 & $3.572(3)$ & 130 \\
\hline
\end{tabular}

Symmetry codes: (i) $x-1, y, z+1$; (ii) $x-1, y, z$; (iii) $x, y, z+1$; (iv) $-x,-y+1,-z+2$. $\operatorname{Cg} A$ and $\operatorname{Cg} B$ are the centroids of the phenyl and pyridine rings respectively.

Data collection: SMART (Bruker, 2001); cell refinement: SAINT (Bruker, 2001); data reduction: $S A I N T$; $\operatorname{program}(\mathrm{s})$ used to solve structure: SHELXS97 (Sheldrick, 2008); program(s) used to refine structure: SHELXL97 (Sheldrick, 2008); molecular graphics: ORTEP-3 (Farrugia, 1997); software used to prepare material for publication: SHELXL97.

We acknowledge the financial support of the National Natural Science Foundation of China (Nos. 20572061 and 20732004) and the Ministry of Science and Technology (No. 2006DFA43030).

Supplementary data and figures for this paper are available from the IUCr electronic archives (Reference: CF2193).

\title{
References
}

Allen, F. H., Kennard, O., Watson, D. G., Brammer, L., Orpen, A. G. \& Taylor, R. (1987). J. Chem. Soc. Perkin Trans. 2, pp. S1-19.

Bruker (2001). SAINT, SMART and SADABS. Bruker AXS Inc., Madison, Wisconsin, USA.

Farrugia, L. J. (1997). J. Appl. Cryst. 30, 565.

Hendrickson, J. B. \& Kandall, C. (1970). Tetrahedron Lett. 5, 343-344.

Pavel, H., Heinrich, L. S. \& Edward, W. S. (1993). J. Am. Chem. Soc. 116, 35003506.

Sheldrick, G. M. (2008). Acta Cryst. A64, 112-122. 


\section{supporting information}

Acta Cryst. (2008). E64, o1104 [doi:10.1107/S1600536808014086]

\section{Benzoylmethyl pyridine-4-carboxylate}

\section{Jian-Nan Guo, Bo-Chao Zhang, Yi Jin, Guo Tang and Yu-Fen Zhao}

\section{S1. Comment}

The title compound was synthesized for a study of protection of the carboxyl group. The phenacyl group has been proved to be an important reagent for protecting carboxyl functions during synthesis in the presence of other esters (Hendrickson et al., 1970).

The title compound (I) was obtained by the reaction of 2-bromo-1-phenylethanone and pyridine-4-carboxylic acid using triethylamine as a base to deprotonate the organic acid. An X-ray crystal structure determination of compound (I) was carried out to determine its conformation. Bond lengths and angles are in agreement with values reported in the literature (Allen et al., 1987). The torsion angle C7-C8-O9-C10 [-80.8 (2) $\AA]$ describes the conformation of the phenyl group with respect to the pyridyl group; the planes of the benzene ring and the pyridine ring form a dihedral angle of 65.8 (1) $\AA$. In the crystal structure of (I), the phenyl and pyridyl rings make a dihedral angle of $65.8(1)^{\circ}$ and the $\mathrm{C} 7-\mathrm{C} 8-\mathrm{O} 9-$ $\mathrm{C} 10$ torsion angle is $-80.8(2)^{\circ}$ (Fig. 1). The packing of the aromatic rings is shown in Fig. 2. Two head-to-tail molecules ( $M$ and $M^{i}$ ) are linked by $\mathrm{C}-\mathrm{H}^{\cdots} \cdots \pi$ interactions with typical geometry (Pavel et al., 1993), leading to the formation of a linear chain. The distance between $\mathrm{CgA}$ and $\mathrm{CgB}^{\mathrm{i}}$ is 6.102 (3) $\AA$ and the angles between the lines through the centroids of the two rings and the normal through $\mathrm{CgA}$ is $79.5(1)^{\circ}$ and through $\mathrm{CgB}^{\mathrm{i}}$ is $76.8(1)^{\circ}$. The linear chains are further stabilized by other $\mathrm{C}-\mathrm{H}^{\cdots} \cdots \pi$ interactions ( $M$ and $M^{\mathrm{ii}} ; M^{\mathrm{i}}$ and $M^{\mathrm{ii}}$ ), generating sheets parallel to $(010)$. The corresponding values for the phenyl ring in $M$ and the pyridyl ring in $M^{\mathrm{ii}}$ are 4.857 (3) $\AA, 69.3(1)^{\circ}$ and $13.7(1)^{\circ}$. For the phenyl ring in $M^{\mathrm{ii}}$ and the pyridyl ring in $M^{\mathrm{i}}$ they are 4.954 (3) $\AA, 69.4(1)^{\circ}$ and $16.6(1)^{\circ} . \mathrm{C}-\mathrm{H}^{\cdots} \pi$ interactions between two sheets $(M$ and $M^{\text {iii) }}$ also provide stability for the crystal structure. The corresponding values for the two adjacent aromatic rings in $\mathrm{M}$ and $\mathrm{M}^{\mathrm{iii}}$ are $4.721(3) \AA, 14.5(1)^{\circ}$ and $66.4(1)^{\circ} . \mathrm{CgA}$ and $\mathrm{CgB}$ stand for the centroids of phenyl and pyridyl rings respectively. [Symmetry codes: (i) $x-1, y, z+1$; (ii) $x-1, y, z$; (iii) $-x, 1-y, 2-z$.]

\section{S2. Experimental}

The title compound was prepared by a method based on one described by Hendrickson \& Kandall (1970). Triethylamine $(1.0 \mathrm{ml}, 7.5 \mathrm{mmol})$ was added dropwise to a mixture of 2-bromo-1-phenylethanone ( $995 \mathrm{mg}, 5 \mathrm{mmol})$ and pyridine-4carboxylic acid $(615 \mathrm{mg}, 5 \mathrm{mmol})$ in freshly distilled tetrahydrofuran $(20 \mathrm{ml})$ at room temperature under argon and stirred overnight. The precipitate was collected at the pump and washed with ethyl acetate. The filtrate and washings were combined and back-washed successively with $1 / 3$ of the volume each of $10 \%$ citric acid, $10 \%$ sodium bicarbonate, and water and then dried. Solvent was distilled off in vacuo and the residue recrystallized repeatedly from ethyl acetatepetroleum ether, giving $1.04 \mathrm{~g}(86 \%)$ as colourless needles.

\section{S3. Refinement}

The hydrogen atoms were positioned geometrically $(\mathrm{C}-\mathrm{H}=0.93,0.98,0.97$ or $0.96 \AA$ for aromatic, tertiary, methylene or methyl $\mathrm{H}$ atoms respectively) and were included in the refinement in the riding model approximation. The 
displacement parameters of methyl $\mathrm{H}$ atoms were set to $1.5 U_{\mathrm{eq}}(\mathrm{C})$, while those of other $\mathrm{H}$ atoms were set to $1.2 U_{\mathrm{eq}}(\mathrm{C})$.

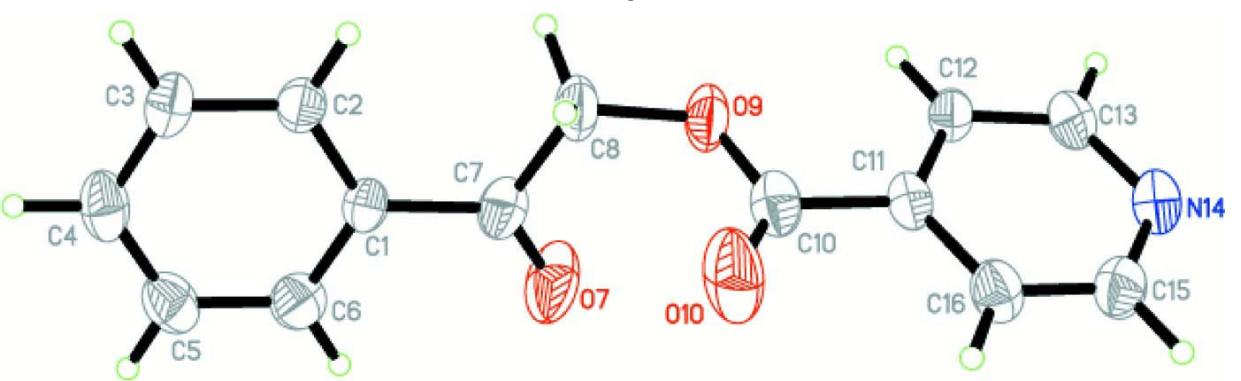

\section{Figure 1}

The molecular structure of (I) with the atom-labelling scheme, showing 50\% probability displacement ellipsoids. $\mathrm{H}$ atoms are drawn as spheres of arbitrary radius.

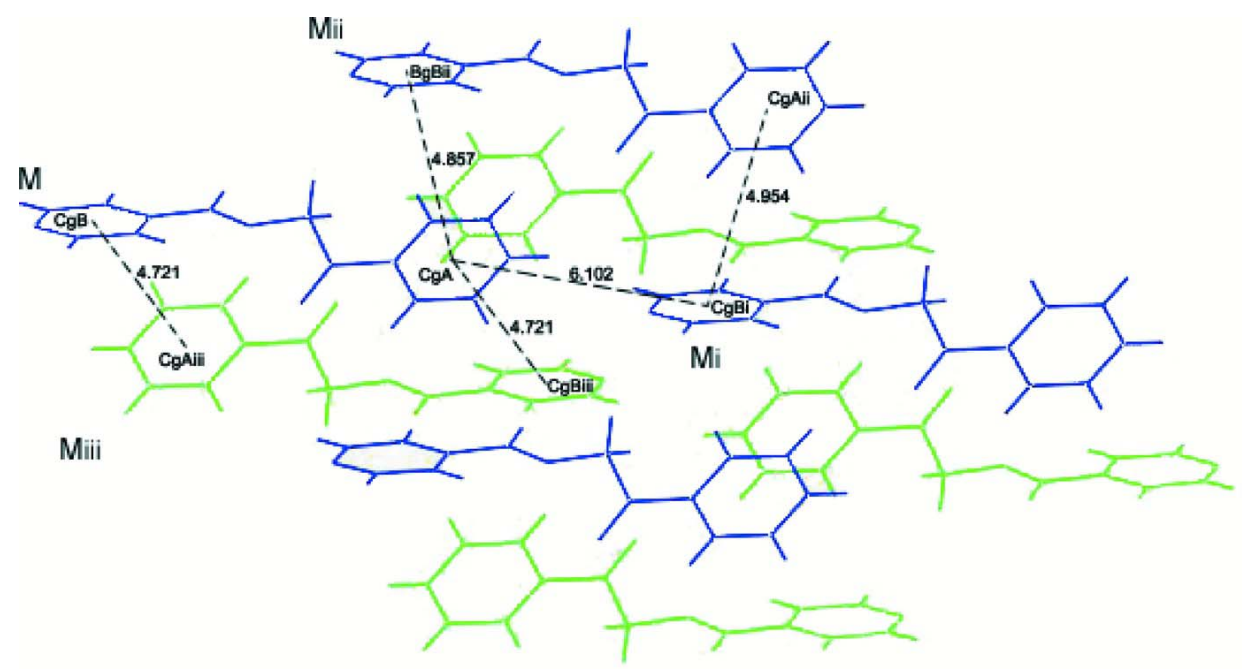

\section{Figure 2}

A view of the $\mathrm{C}-\mathrm{H}^{\cdots} \pi \pi$ interactions motif of (I). $\mathrm{CgA}$ and $\mathrm{CgB}$ are the centroids of the benzene and pyridine rings respectively. The $\mathrm{C}-\mathrm{H} \cdots \pi$ interactions are shown as dashed lines. The molecules labelled with same color are in one plane. [Symmetry code: (i) x-1, y, z+1; (ii) x-1, y, z; (iii) -x, 1-y, 2-z.]"

\section{Benzoylmethyl pyridine-4-carboxylate}

Crystal data

$\mathrm{C}_{14} \mathrm{H}_{11} \mathrm{NO}_{3}$

$M_{r}=241.24$

Triclinic, $P \overline{1}$

Hall symbol: -P 1

$a=8.0863(6) \AA$

$b=9.2130(7) \AA$

$c=9.3291(8) \AA$

$\alpha=106.738(7)^{\circ}$

$\beta=114.495(8)^{\circ}$

$\gamma=96.549(6)^{\circ}$

$V=583.52(8) \AA^{3}$
$Z=2$

$F(000)=252$

$D_{\mathrm{x}}=1.373 \mathrm{Mg} \mathrm{m}^{-3}$

Mo $K \alpha$ radiation, $\lambda=0.71073 \AA$

Cell parameters from 1153 reflections

$\theta=2.4-32.7^{\circ}$

$\mu=0.10 \mathrm{~mm}^{-1}$

$T=173 \mathrm{~K}$

Needle, colorless

$0.30 \times 0.17 \times 0.12 \mathrm{~mm}$ 


\section{Data collection}

Bruker APEX CCD

diffractometer

Radiation source: fine-focus sealed tube

Graphite monochromator

Detector resolution: 16.1903 pixels $\mathrm{mm}^{-1}$

$\varphi$ and $\omega$ scans

Absorption correction: multi-scan

(SADABS; Bruker, 2001)

$T_{\min }=0.971, T_{\max }=0.988$

\section{Refinement}

Refinement on $F^{2}$

Least-squares matrix: full

$R\left[F^{2}>2 \sigma\left(F^{2}\right)\right]=0.039$

$w R\left(F^{2}\right)=0.074$

$S=0.82$

2011 reflections

163 parameters

0 restraints

Primary atom site location: structure-invariant direct methods
4945 measured reflections

2011 independent reflections

1099 reflections with $I>2 \sigma(I)$

$R_{\text {int }}=0.042$

$\theta_{\max }=25.0^{\circ}, \theta_{\min }=2.4^{\circ}$

$h=-9 \rightarrow 9$

$k=-10 \rightarrow 9$

$l=-10 \rightarrow 11$

Secondary atom site location: difference Fourier map

Hydrogen site location: inferred from

neighbouring sites

$\mathrm{H}$-atom parameters constrained

$w=1 /\left[\sigma^{2}\left(F_{\mathrm{o}}^{2}\right)+(0.0282 P)^{2}\right]$

where $P=\left(F_{\mathrm{o}}^{2}+2 F_{\mathrm{c}}^{2}\right) / 3$

$(\Delta / \sigma)_{\max }<0.001$

$\Delta \rho_{\max }=0.11$ e $\AA^{-3}$

$\Delta \rho_{\text {min }}=-0.13$ e $\AA^{-3}$

\section{Special details}

Geometry. All e.s.d.'s (except the e.s.d. in the dihedral angle between two 1.s. planes) are estimated using the full covariance matrix. The cell e.s.d.'s are taken into account individually in the estimation of e.s.d.'s in distances, angles and torsion angles; correlations between e.s.d.'s in cell parameters are only used when they are defined by crystal symmetry. An approximate (isotropic) treatment of cell e.s.d.'s is used for estimating e.s.d.'s involving 1.s. planes.

Refinement. Refinement of $F^{2}$ against ALL reflections. The weighted $R$-factor $w R$ and goodness of fit $S$ are based on $F^{2}$, conventional $R$-factors $R$ are based on $F$, with $F$ set to zero for negative $F^{2}$. The threshold expression of $F^{2}>\sigma\left(F^{2}\right)$ is used only for calculating $R$-factors $(\mathrm{gt})$ etc. and is not relevant to the choice of reflections for refinement. $R$-factors based on $F^{2}$ are statistically about twice as large as those based on $F$, and $R$-factors based on ALL data will be even larger.

Fractional atomic coordinates and isotropic or equivalent isotropic displacement parameters $\left(\AA^{2}\right)$

\begin{tabular}{lllll}
\hline & $x$ & $y$ & $z$ & $U_{\text {iso }} * / U_{\text {eq }}$ \\
\hline C1 & $-0.1339(2)$ & $0.7322(2)$ & $1.1693(2)$ & $0.0306(5)$ \\
C2 & $-0.3294(2)$ & $0.6903(2)$ & $1.0760(2)$ & $0.0329(5)$ \\
H2 & -0.3880 & 0.6499 & 0.9555 & $0.039 *$ \\
C3 & $-0.4385(2)$ & $0.7073(2)$ & $1.1574(3)$ & $0.0359(5)$ \\
H3 & -0.5721 & 0.6780 & 1.0931 & $0.043^{*}$ \\
C4 & $-0.3540(3)$ & $0.7668(2)$ & $1.3323(3)$ & $0.0412(5)$ \\
H4 & -0.4296 & 0.7781 & 1.3881 & $0.049 *$ \\
C5 & $-0.1609(3)$ & $0.8098(2)$ & $1.4261(3)$ & $0.0428(5)$ \\
H5 & -0.1030 & 0.8523 & 1.5465 & $0.051^{*}$ \\
C6 & $-0.0511(3)$ & $0.7910(2)$ & $1.3450(2)$ & $0.0395(5)$ \\
H6 & 0.0823 & 0.8186 & 1.4101 & $0.047 *$ \\
C7 & $-0.0110(3)$ & $0.7152(2)$ & $1.0855(3)$ & $0.0382(5)$ \\
O7 & $0.15653(19)$ & $0.7341(2)$ & $1.16374(19)$ & $0.0670(5)$ \\
C8 & $-0.1064(2)$ & $0.6727(3)$ & $0.8971(2)$ & $0.0435(6)$ \\
H8B & -0.2071 & 0.5732 & 0.8425 & $0.052^{*}$ \\
H8A & -0.1661 & 0.7559 & 0.8701 & $0.052^{*}$
\end{tabular}


supporting information

\begin{tabular}{lllll} 
O9 & $0.02347(17)$ & $0.65426(17)$ & $0.82904(17)$ & $0.0437(4)$ \\
C10 & $0.1269(3)$ & $0.7883(3)$ & $0.8448(3)$ & $0.0385(5)$ \\
O10 & $0.1180(2)$ & $0.91629(19)$ & $0.9139(2)$ & $0.0649(5)$ \\
C11 & $0.2487(3)$ & $0.7577(3)$ & $0.7621(2)$ & $0.0313(5)$ \\
C12 & $0.2426(2)$ & $0.6074(2)$ & $0.6700(2)$ & $0.0320(5)$ \\
H12 & 0.1609 & 0.5172 & 0.6585 & $0.038^{*}$ \\
C13 & $0.3593(2)$ & $0.5930(2)$ & $0.5953(2)$ & $0.0364(5)$ \\
H13 & 0.3537 & 0.4900 & 0.5309 & $0.044^{*}$ \\
N14 & $0.4789(2)$ & $0.7134(2)$ & $0.6074(2)$ & $0.0396(5)$ \\
C15 & $0.4825(3)$ & $0.8565(3)$ & $0.6977(3)$ & $0.0420(5)$ \\
H15 & 0.5670 & 0.9447 & 0.7090 & $0.050^{*}$ \\
C16 & $0.3708(3)$ & $0.8837(2)$ & $0.7753(2)$ & $0.0383(5)$ \\
H16 & 0.3779 & 0.9881 & 0.8372 & $0.046^{*}$ \\
\hline
\end{tabular}

Atomic displacement parameters $\left(\AA^{2}\right)$

\begin{tabular}{lllllll}
\hline & $U^{11}$ & $U^{22}$ & $U^{33}$ & $U^{12}$ & $U^{13}$ & $U^{23}$ \\
\hline C1 & $0.0319(10)$ & $0.0331(13)$ & $0.0341(12)$ & $0.0088(9)$ & $0.0194(9)$ & $0.0165(11)$ \\
C2 & $0.0308(10)$ & $0.0375(13)$ & $0.0332(12)$ & $0.0093(10)$ & $0.0170(10)$ & $0.0140(11)$ \\
C3 & $0.0365(11)$ & $0.0362(13)$ & $0.0503(14)$ & $0.0156(10)$ & $0.0285(11)$ & $0.0220(12)$ \\
C4 & $0.0579(13)$ & $0.0397(14)$ & $0.0509(15)$ & $0.0211(12)$ & $0.0400(12)$ & $0.0257(13)$ \\
C5 & $0.0564(14)$ & $0.0438(14)$ & $0.0335(12)$ & $0.0140(12)$ & $0.0244(12)$ & $0.0165(12)$ \\
C6 & $0.0389(12)$ & $0.0429(14)$ & $0.0373(13)$ & $0.0097(11)$ & $0.0170(11)$ & $0.0176(12)$ \\
C7 & $0.0326(11)$ & $0.0475(14)$ & $0.0450(13)$ & $0.0155(11)$ & $0.0221(11)$ & $0.0238(12)$ \\
O7 & $0.0326(8)$ & $0.1257(16)$ & $0.0589(10)$ & $0.0276(10)$ & $0.0249(8)$ & $0.0486(12)$ \\
C8 & $0.0364(11)$ & $0.0638(16)$ & $0.0409(13)$ & $0.0164(12)$ & $0.0272(11)$ & $0.0188(13)$ \\
O9 & $0.0439(8)$ & $0.0525(10)$ & $0.0496(9)$ & $0.0144(8)$ & $0.0353(8)$ & $0.0186(9)$ \\
C10 & $0.0416(12)$ & $0.0492(15)$ & $0.0369(13)$ & $0.0197(12)$ & $0.0232(11)$ & $0.0225(13)$ \\
O10 & $0.0978(12)$ & $0.0517(11)$ & $0.0899(13)$ & $0.0419(10)$ & $0.0749(11)$ & $0.0320(11)$ \\
C11 & $0.0336(10)$ & $0.0400(12)$ & $0.0291(11)$ & $0.0155(10)$ & $0.0188(9)$ & $0.0163(11)$ \\
C12 & $0.0328(10)$ & $0.0362(13)$ & $0.0321(11)$ & $0.0091(10)$ & $0.0192(10)$ & $0.0133(11)$ \\
C13 & $0.0422(11)$ & $0.0408(14)$ & $0.0342(12)$ & $0.0177(11)$ & $0.0229(10)$ & $0.0144(11)$ \\
N14 & $0.0452(10)$ & $0.0467(13)$ & $0.0401(11)$ & $0.0170(10)$ & $0.0269(9)$ & $0.0216(10)$ \\
C15 & $0.0492(12)$ & $0.0411(14)$ & $0.0425(13)$ & $0.0081(12)$ & $0.0264(12)$ & $0.0188(12)$ \\
C16 & $0.0501(12)$ & $0.0354(13)$ & $0.0390(12)$ & $0.0159(11)$ & $0.0274(11)$ & $0.0153(11)$ \\
& & & & & & \\
\hline
\end{tabular}

Geometric parameters $\left(\AA,{ }^{\circ}\right)$

\begin{tabular}{llll}
\hline $\mathrm{C} 1-\mathrm{C} 6$ & $1.387(2)$ & $\mathrm{C} 8-\mathrm{H} 8 \mathrm{~B}$ & 0.990 \\
$\mathrm{C} 1-\mathrm{C} 2$ & $1.390(2)$ & $\mathrm{C} 8-\mathrm{H} 8 \mathrm{~A}$ & 0.990 \\
$\mathrm{C} 1-\mathrm{C} 7$ & $1.495(2)$ & $\mathrm{O}-\mathrm{C} 10$ & $1.343(2)$ \\
$\mathrm{C} 2-\mathrm{C} 3$ & $1.378(2)$ & $\mathrm{C} 10-\mathrm{O} 10$ & $1.196(2)$ \\
$\mathrm{C} 2-\mathrm{H} 2$ & 0.950 & $\mathrm{C} 10-\mathrm{C} 11$ & $1.488(2)$ \\
$\mathrm{C} 3-\mathrm{C} 4$ & $1.380(3)$ & $\mathrm{C} 11-\mathrm{C} 16$ & $1.376(2)$ \\
$\mathrm{C} 3-\mathrm{H} 3$ & 0.950 & $\mathrm{C} 11-\mathrm{C} 12$ & $1.386(3)$ \\
$\mathrm{C} 4-\mathrm{C} 5$ & $1.374(3)$ & $\mathrm{C} 12-\mathrm{C} 13$ & $1.384(2)$ \\
$\mathrm{C} 4-\mathrm{H} 4$ & 0.950 & $\mathrm{C} 12-\mathrm{H} 12$ & 0.950 \\
$\mathrm{C} 5-\mathrm{C} 6$ & $1.381(2)$ & $\mathrm{C} 13-\mathrm{N} 14$ & $1.330(2)$
\end{tabular}




\begin{tabular}{|c|c|c|c|}
\hline $\mathrm{C} 5-\mathrm{H} 5$ & 0.950 & C13-H13 & 0.950 \\
\hline $\mathrm{C} 6-\mathrm{H} 6$ & 0.950 & $\mathrm{~N} 14-\mathrm{C} 15$ & $1.334(2)$ \\
\hline $\mathrm{C} 7-\mathrm{O} 7$ & $1.204(2)$ & $\mathrm{C} 15-\mathrm{C} 16$ & $1.375(2)$ \\
\hline $\mathrm{C} 7-\mathrm{C} 8$ & $1.499(3)$ & C15-H15 & 0.950 \\
\hline $\mathrm{C} 8-\mathrm{O} 9$ & $1.4371(19)$ & $\mathrm{C} 16-\mathrm{H} 16$ & 0.950 \\
\hline $\mathrm{C} 6-\mathrm{C} 1-\mathrm{C} 2$ & $118.97(16)$ & $\mathrm{O} 9-\mathrm{C} 8-\mathrm{H} 8 \mathrm{~A}$ & 109.2 \\
\hline $\mathrm{C} 6-\mathrm{C} 1-\mathrm{C} 7$ & $119.22(17)$ & $\mathrm{C} 7-\mathrm{C} 8-\mathrm{H} 8 \mathrm{~A}$ & 109.2 \\
\hline $\mathrm{C} 2-\mathrm{C} 1-\mathrm{C} 7$ & $121.80(17)$ & $\mathrm{H} 8 \mathrm{~B}-\mathrm{C} 8-\mathrm{H} 8 \mathrm{~A}$ & 107.9 \\
\hline $\mathrm{C} 3-\mathrm{C} 2-\mathrm{C} 1$ & $120.25(18)$ & $\mathrm{C} 10-\mathrm{O} 9-\mathrm{C} 8$ & $115.55(15)$ \\
\hline $\mathrm{C} 3-\mathrm{C} 2-\mathrm{H} 2$ & 119.9 & $\mathrm{O} 10-\mathrm{C} 10-\mathrm{O} 9$ & $123.68(17)$ \\
\hline $\mathrm{C} 1-\mathrm{C} 2-\mathrm{H} 2$ & 119.9 & $\mathrm{O} 10-\mathrm{C} 10-\mathrm{C} 11$ & $124.4(2)$ \\
\hline $\mathrm{C} 2-\mathrm{C} 3-\mathrm{C} 4$ & $120.10(18)$ & $\mathrm{O} 9-\mathrm{C} 10-\mathrm{C} 11$ & $111.85(18)$ \\
\hline $\mathrm{C} 2-\mathrm{C} 3-\mathrm{H} 3$ & 119.9 & $\mathrm{C} 16-\mathrm{C} 11-\mathrm{C} 12$ & $118.45(16)$ \\
\hline $\mathrm{C} 4-\mathrm{C} 3-\mathrm{H} 3$ & 119.9 & $\mathrm{C} 16-\mathrm{C} 11-\mathrm{C} 10$ & 118.77 (19) \\
\hline $\mathrm{C} 5-\mathrm{C} 4-\mathrm{C} 3$ & $120.23(17)$ & $\mathrm{C} 12-\mathrm{C} 11-\mathrm{C} 10$ & 122.77 (19) \\
\hline $\mathrm{C} 5-\mathrm{C} 4-\mathrm{H} 4$ & 119.9 & $\mathrm{C} 13-\mathrm{C} 12-\mathrm{C} 11$ & $117.82(17)$ \\
\hline $\mathrm{C} 3-\mathrm{C} 4-\mathrm{H} 4$ & 119.9 & $\mathrm{C} 13-\mathrm{C} 12-\mathrm{H} 12$ & 121.1 \\
\hline $\mathrm{C} 4-\mathrm{C} 5-\mathrm{C} 6$ & $119.85(19)$ & $\mathrm{C} 11-\mathrm{C} 12-\mathrm{H} 12$ & 121.1 \\
\hline $\mathrm{C} 4-\mathrm{C} 5-\mathrm{H} 5$ & 120.1 & $\mathrm{~N} 14-\mathrm{C} 13-\mathrm{C} 12$ & $124.53(18)$ \\
\hline $\mathrm{C} 6-\mathrm{C} 5-\mathrm{H} 5$ & 120.1 & $\mathrm{~N} 14-\mathrm{C} 13-\mathrm{H} 13$ & 117.7 \\
\hline $\mathrm{C} 5-\mathrm{C} 6-\mathrm{C} 1$ & $120.57(18)$ & $\mathrm{C} 12-\mathrm{C} 13-\mathrm{H} 13$ & 117.7 \\
\hline $\mathrm{C} 5-\mathrm{C} 6-\mathrm{H} 6$ & 119.7 & $\mathrm{C} 13-\mathrm{N} 14-\mathrm{C} 15$ & $116.29(15)$ \\
\hline $\mathrm{C} 1-\mathrm{C} 6-\mathrm{H} 6$ & 119.7 & $\mathrm{~N} 14-\mathrm{C} 15-\mathrm{C} 16$ & $123.75(19)$ \\
\hline $\mathrm{O} 7-\mathrm{C} 7-\mathrm{C} 1$ & $122.35(18)$ & N14-C15-H15 & 118.1 \\
\hline $\mathrm{O} 7-\mathrm{C} 7-\mathrm{C} 8$ & $120.97(16)$ & $\mathrm{C} 16-\mathrm{C} 15-\mathrm{H} 15$ & 118.1 \\
\hline $\mathrm{C} 1-\mathrm{C} 7-\mathrm{C} 8$ & $116.67(15)$ & $\mathrm{C} 15-\mathrm{C} 16-\mathrm{C} 11$ & $119.15(18)$ \\
\hline $\mathrm{O} 9-\mathrm{C} 8-\mathrm{C} 7$ & $112.06(15)$ & $\mathrm{C} 15-\mathrm{C} 16-\mathrm{H} 16$ & 120.4 \\
\hline $\mathrm{O} 9-\mathrm{C} 8-\mathrm{H} 8 \mathrm{~B}$ & 109.2 & $\mathrm{C} 11-\mathrm{C} 16-\mathrm{H} 16$ & 120.4 \\
\hline $\mathrm{C} 7-\mathrm{C} 8-\mathrm{H} 8 \mathrm{~B}$ & 109.2 & & \\
\hline $\mathrm{C} 6-\mathrm{C} 1-\mathrm{C} 2-\mathrm{C} 3$ & $0.0(3)$ & $\mathrm{C} 8-\mathrm{O} 9-\mathrm{C} 10-\mathrm{O} 10$ & $1.7(3)$ \\
\hline $\mathrm{C} 7-\mathrm{C} 1-\mathrm{C} 2-\mathrm{C} 3$ & $179.89(17)$ & $\mathrm{C} 8-\mathrm{O} 9-\mathrm{C} 10-\mathrm{C} 11$ & $-176.62(16)$ \\
\hline $\mathrm{C} 1-\mathrm{C} 2-\mathrm{C} 3-\mathrm{C} 4$ & $0.3(3)$ & $\mathrm{O} 10-\mathrm{C} 10-\mathrm{C} 11-\mathrm{C} 16$ & $5.1(3)$ \\
\hline $\mathrm{C} 2-\mathrm{C} 3-\mathrm{C} 4-\mathrm{C} 5$ & $0.2(3)$ & $\mathrm{O} 9-\mathrm{C} 10-\mathrm{C} 11-\mathrm{C} 16$ & $-176.53(18)$ \\
\hline $\mathrm{C} 3-\mathrm{C} 4-\mathrm{C} 5-\mathrm{C} 6$ & $-1.0(3)$ & $\mathrm{O} 10-\mathrm{C} 10-\mathrm{C} 11-\mathrm{C} 12$ & $-173.9(2)$ \\
\hline $\mathrm{C} 4-\mathrm{C} 5-\mathrm{C} 6-\mathrm{C} 1$ & $1.4(3)$ & $\mathrm{O} 9-\mathrm{C} 10-\mathrm{C} 11-\mathrm{C} 12$ & $4.4(3)$ \\
\hline $\mathrm{C} 2-\mathrm{C} 1-\mathrm{C} 6-\mathrm{C} 5$ & $-0.9(3)$ & $\mathrm{C} 16-\mathrm{C} 11-\mathrm{C} 12-\mathrm{C} 13$ & $-0.5(3)$ \\
\hline $\mathrm{C} 7-\mathrm{C} 1-\mathrm{C} 6-\mathrm{C} 5$ & $179.23(19)$ & $\mathrm{C} 10-\mathrm{C} 11-\mathrm{C} 12-\mathrm{C} 13$ & $178.58(17)$ \\
\hline $\mathrm{C} 6-\mathrm{C} 1-\mathrm{C} 7-\mathrm{O} 7$ & $8.4(3)$ & $\mathrm{C} 11-\mathrm{C} 12-\mathrm{C} 13-\mathrm{N} 14$ & $0.9(3)$ \\
\hline $\mathrm{C} 2-\mathrm{C} 1-\mathrm{C} 7-\mathrm{O} 7$ & $-171.5(2)$ & $\mathrm{C} 12-\mathrm{C} 13-\mathrm{N} 14-\mathrm{C} 15$ & $-0.5(3)$ \\
\hline $\mathrm{C} 6-\mathrm{C} 1-\mathrm{C} 7-\mathrm{C} 8$ & $-171.7(2)$ & $\mathrm{C} 13-\mathrm{N} 14-\mathrm{C} 15-\mathrm{C} 16$ & $-0.3(3)$ \\
\hline $\mathrm{C} 2-\mathrm{C} 1-\mathrm{C} 7-\mathrm{C} 8$ & $8.4(3)$ & $\mathrm{N} 14-\mathrm{C} 15-\mathrm{C} 16-\mathrm{C} 11$ & $0.6(3)$ \\
\hline $\mathrm{O} 7-\mathrm{C} 7-\mathrm{C} 8-\mathrm{O} 9$ & $1.8(3)$ & $\mathrm{C} 12-\mathrm{C} 11-\mathrm{C} 16-\mathrm{C} 15$ & $-0.2(3)$ \\
\hline $\mathrm{C} 1-\mathrm{C} 7-\mathrm{C} 8-\mathrm{O} 9$ & $-178.06(16)$ & $\mathrm{C} 10-\mathrm{C} 11-\mathrm{C} 16-\mathrm{C} 15$ & $-179.31(19)$ \\
\hline $\mathrm{C} 7-\mathrm{C} 8-\mathrm{O} 9-\mathrm{C} 10$ & $-80.8(2)$ & & \\
\hline
\end{tabular}




\section{supporting information}

Hydrogen-bond geometry $\left(\AA,{ }^{\circ}\right)$

\begin{tabular}{lllll}
\hline$D-\mathrm{H} \cdots A$ & $D-\mathrm{H}$ & $\mathrm{H} \cdots A$ & $D \cdots A$ & $D-\mathrm{H} \cdots A$ \\
\hline $\mathrm{C} 4-\mathrm{H} 4 \cdots \mathrm{CgB}^{\mathrm{i}}$ & 0.95 & 3.86 & $4.766(3)$ & 160 \\
$\mathrm{C} 2-\mathrm{H} 2 \cdots \mathrm{CgB}^{\mathrm{ii}}$ & 0.95 & 2.89 & $3.640(3)$ & 137 \\
$\mathrm{C} 6-\mathrm{H} 6 \cdots \mathrm{CgB}^{\text {iii }}$ & 0.95 & 3.00 & $3.752(3)$ & 137 \\
$\mathrm{C} 12-\mathrm{H} 12 \cdots \mathrm{CgA}^{\mathrm{iv}}$ & 0.95 & 2.88 & $3.572(3)$ & 130
\end{tabular}

Symmetry codes: (i) $x-1, y, z+1$; (ii) $x-1, y, z$; (iii) $x, y, z+1$; (iv) $-x,-y+1,-z+2$. 